\title{
Unsupported Pt Nanoparticles: Synthesis, Deactivation, and Hydrogen Electrocatalysis in Unpurified Electrolytes
}

\author{
Rituja B. Patil, Qiudi Meng, and James R. McKone* \\ Department of Chemical and Petroleum Engineering, University of Pittsburgh, Pittsburgh, PA, \\ 15260, USA \\ E-mail: jmckone@pitt.edu* \\ Phone: +1 (412)-384-7407
}

\begin{abstract}
Platinum is ubiquitous in electrochemical catalysis owing to its ability to accelerate redox reactions involving surface-bound hydrogen and oxygen. Accordingly, Pt is often used as a calibration standard and activity benchmark against which novel electrocatalysts are compared. These measurements are often executed in unpurified, reagent grade electrolytes where Pt is also susceptible to deactivation by several routes. This constitutes a challenge where the ease with which Pt-based electrocatalysis measurements can be executed must be balanced against the difficulty of making those measurements accurate and consistent. We report herein a synthetic procedure for catalytically active Pt nanoparticles that uses readily available reagents and laboratory apparatus, with the goal of making high-quality control experiments in electrocatalysis as easy as possible. We also identified conditions under which these particles deactivate in unpurified aqueous acid and base and differentiated between mechanisms involving catalyst poisoning, which dominates at more negative applied potentials, and particle growth, which dominates at positive potentials where Pt-oxide species are produced. Finally, we demonstrated that unsupported Pt nanoparticle films can be used to good effect for reference electrode cali-
\end{abstract}


bration and benchmarking of hydrogen evolution/oxidation electrocatalysts, even in unpurified electrolytes, provided steps are taken to minimize the impact of deactivation.

\section{Keywords}

platinum; synthesis; nanoparticles; deactivation; ECSA; IL-TEM

\section{Introduction}

Platinum is one of the most widely studied transition metals for applications in thermal and electrochemical catalysis. ${ }^{1-[5}$ The many thermocatalytic applications of Pt include automotive exhaust treatment $^{6+9}$ as well as the production of nitric acid, ${ }^{10,11}$ silicones, $\frac{12,13}{2}$ and petrochemical feedstocks. ${ }^{14 \cdot 15} \mathrm{Pt}$ is also used ubiquitously in analytical and industrial electrochemistry, and many of these applications revolve around its high catalytic activity toward reactions involving hydrogen. ${ }^{16-25}$ For example, carbon-supported Pt catalysts $(\mathrm{Pt} / \mathrm{C})$ are used for the hydrogen evolution reaction (HER) in proton-exchange membrane electrolyzers and the hydrogen oxidation reaction (HOR) in low and intermediate temperature fuel cells. ${ }^{26}$ Despite its very high catalytic activity, Pt is susceptible to deactivation under electrochemical conditions; thus, the dynamics and mechanisms of Pt deactivation have also been studied extensively. ${ }^{27.31}$ The most prevalent deactivation processes generally fall in one of several categories: catalyst poisoning, particle growth, particle detachment, and dissolution. Ultimately, the dynamics of these deactivation processes dictate the practical lifetime of Pt catalysts. Thus, a major research focus on Pt has been on evaluating and minimizing the impact of catalyst deactivation in analytical and practical applications. $.32+35$

Owing to its very high catalytic activity for hydrogen evolution/oxidation electrocatalysis, Pt is used as a benchmark against which novel HER/HOR catalysts are measured. It is also used to calibrate voltage measurements in aqueous electrocatalysis via the determination of the reversible hydrogen electrode (RHE) potential in the electrolyte of interest. The propensity of Pt electrocatalysts to deactivate under reaction conditions makes these key measurements considerably more challeng- 
ing. $\frac{36}{38}$ Whereas prior studies have generally addressed this challenge by maintaining scrupulously

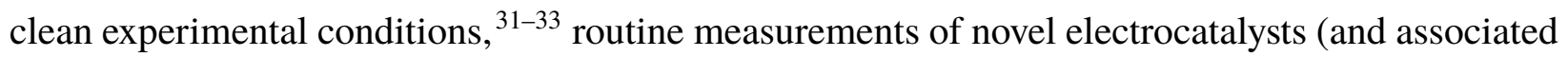
comparisons to $\mathrm{Pt}$ ) are often carried out in unpurified, reagent-grade electrolytes. This motivates the question of whether control experiments using Pt electrodes can be properly executed.

Herein, we describe a straightforward preparation of unsupported Pt nanoparticles (NPs) as well as their use as a laboratory standard for hydrogen evolution/oxidation studies. We have focused on experimental conditions that are not scrupulously clean but are nevertheless representative of the methods and materials used for routine measurements in applied electrocatalysis research laboratories. Accordingly, we also studied the deactivation of Pt NPs, as reflected in the progressive loss of electrochemically active surface area. When cycled continuously between oxidizing and reducing conditions in unpurified aqueous acidic and alkaline electrolytes, our Pt NPs lost 25-40 \% of their active surface area over tens of minutes. We also deployed identical location transmission elec-

tron microscopy (IL-TEM) ${ }^{39 \mid 40}$ to differentiate deactivation processes involving changes in catalyst morphology from those that do not. At positive potential limits below $1 \mathrm{~V}$ vs RHE, we observed minimal changes in particle size/shape, implying the dominant mode of deactivation is catalyst poisoning rather than particle dissolution or growth. These observations were found to be consistent across acid and base conditions, but the relative rate of deactivation were modestly slower in base than in acid. By contrast, when the positive potential limit was increased to $1.5 \mathrm{~V}$ vs RHE, the Pt NPs were found to grow in size, leading to irreversible loss in surface area.

\section{Experimental section}

The synthesis protocol for Pt nanoparticles was developed from several prior literature reports. 41,43 A representative procedure is as follows. Aqueous stock solutions of $12 \mathrm{mM} \mathrm{H}_{2} \mathrm{PtCl}_{6}(99.5 \%$ Alfa Aesar) precursor and $100 \mathrm{mM}$ sodium polyacrylate (average molecular weight $\sim 1200,45 \mathrm{wt} \%$ in water, 99.5\%, Alfa Aesar), respectively, were prepared by dissolution with ultrasonic agitation for at least 5 minutes. These solutions were prepared in bulk quantities and used for several synthetic 
runs. A fresh solution of $100 \mathrm{mM}$ L-ascorbic acid reducing agent was prepared immediately before the synthesis by adding $0.0195 \mathrm{~g}$ of anhydrous $\mathrm{L}-\mathrm{C}_{6} \mathrm{H}_{8} \mathrm{O}_{6}(99.5 \%$, Alfa Aesar) to $1 \mathrm{~mL}$ deionized water and mixed by sonication. In a $20 \mathrm{~mL}$ scintiallation vial, $0.833 \mathrm{~g}$ of $12 \mathrm{mM} \mathrm{H}_{2} \mathrm{PtCl}_{6}$ and $0.1 \mathrm{~g}$ of $100 \mathrm{mM}$ sodium polyacrylate were added to $1 \mathrm{~mL}$ ascorbic acid solution. deionized water $(\geq 18$ $\mathrm{M} \Omega \mathrm{cm}$ resistivity, Millipore) was then added to increase the solution volume to $10 \mathrm{ml}$. The final concentrations of the reagents in the precursor solution were $1 \mathrm{mM}$ each of $\mathrm{H}_{2} \mathrm{PtCl}_{6}$ and sodium polyacrylate and $10 \mathrm{mM}$ ascorbic acid. The precursor mixture was initially transparent with a slight yellow color.

The vial was placed in a preheated bath of silicone oil (Fluke 5010, Type 200.05) at $90{ }^{\circ} \mathrm{C}$. The contents were stirred at $600 \mathrm{rpm}$ for 60 minutes using a magnetic stir bar. The temperature was monitored using an alcohol thermometer to ensure that the variation was within $\pm 3{ }^{\circ} \mathrm{C}$. Within 15 minutes, the solution became black and opaque. The reaction was allowed to continue for 60 minutes, and successive additions of deionized water were made every 20 minutes to maintain a constant total volume of solution. The vial was then removed from the bath and allowed to cool at room temperature. After cooling, $0.4 \mathrm{~g} \mathrm{NaOH}$ (98\%, Alfa Aesar) was added to the suspension to alkalize the solution to $\mathrm{pH} \sim 14$. The vial was then capped and left to stand at room temperature for 14 days, during which the black suspended solids settled to the bottom of the vial. Note that this settling process was sometimes found to proceed over a shorter timescale, but a 14-day incubation period was always found to be sufficient for particles to settle.

The clear solution above the settled layer was decanted using a pipette and the particles were extracted by centrifugation at $6000 \mathrm{rpm}$ for 15 minutes. The supernatant was again decanted and the particles were re-suspended and centrifuged using $1 \mathrm{M}$ aqueous $\mathrm{NaOH}$ solution a total of 3 times. Finally, the particles were suspended in $1.5 \mathrm{ml}$ of deoinized water; this suspension was found to remain stable for several days. The colloidal solution was either used as-is or further diluted with pure deionized water as appropriate prior to subsequent analysis.

The NP yield was measured by depositing a known volume of the final colloid on a pre-weighed borosilicate glass microscope slide. The particles were dried under an infrared heat lamp (McMas- 
ter Carr, 125 watt, BR40 type bulb) for $\sim 45$ minutes. The mass of the glass slide with the dried film of Pt nanoparticles was again recorded. The particle yield was thus calculated by subtracting the mass of the initial uncoated slide from the Pt-coated slide. The average yield obtained after the centrifugation process from 6 synthetic runs was $1.48 \pm 0.03 \mathrm{mg}$, which is $76 \pm 1 \%$ of the 1.95 mg theoretical yield. Note that this error bound, and all others unless otherwise noted, represent 1.96 times the standard error of the mean, which we interpret as a $95 \%$ confidence interval.

The final, purified Pt NPs were characterized with respect to composition and morphology using Fourier-transform infrared spectroscopy (FTIR), X-ray diffraction (XRD), and transmission electron microscopy (TEM). FTIR spectra were collected on Bruker VERTEX-70LS instrument using an attenuated total reflection (ATR) accessory at a resolution of $4 \mathrm{~cm}^{-1}$. Deionized water was used as a blank solution, with aqueous Pt NP suspension as the experimental sample. A Bruker D8 system with $\mathrm{Cu} \mathrm{K} \alpha$ radiation $(\lambda=1.54 \AA$ ) was used for XRD analysis. Diffraction patterns were collected from $30^{\circ}$ to $90^{\circ} 2 \theta$ with $0.02^{\circ}$ steps. The data collection time per step was optimized empirically to yield diffraction peaks that were well resolved from the noise floor.

Transmission electron microscopy (TEM) imaging was executed using a Hitachi H-9500 environmental transmission electron microscope at an accelerating voltage of $300 \mathrm{kV}$. For IL-TEM analysis, the nanoparticles were deposited on center-marked 400 mesh Au TEM grids (Ted Pella). A perforation was made manually near the edge of the grid using sharp tweezers (Ted Pella). Approximately $5 \mu \mathrm{L}$ of the Pt nanoparticle suspension was dropcast on the grid and dried under an infrared heat lamp. The dried grid was then immersed in water to remove soluble impurities and poorly adhered NPs. Finally, a hooked gold wire (0.5 mm, 99.999\%, Fisher Scientific) was inserted through the perforation to make electrical contact for electrochemical measurements.

A Gamry Reference 600+ Potentiostat was used to characterized the electrochemical properties of the Pt nanoparticles. For non-TEM based studies, $10 \mu \mathrm{L}$ of a known loading of platinum suspension was dropcast onto a $3 \mathrm{~mm}$ diameter $\left(0.071 \mathrm{~cm}^{2}\right.$ surface area) glassy carbon electrode and dried under infrared. The counter electrode was a Pt wire in acidic electrolyte and Ni foil in alkaline conditions; the reference electrode was $\mathrm{Ag} / \mathrm{AgCl}(1 \mathrm{M} \mathrm{KCl})$. The potential from the $\mathrm{Ag} / \mathrm{AgCl}$ reference 
electrode was later converted to the reversible hydrogen electrode (RHE) potential by measuring the open circuit potential value of a clean Pt NP film in $\mathrm{H}_{2}$-saturated electrolyte at $1 \mathrm{~atm}$. Electrolytes used for electrochemical analysis were $0.5 \mathrm{M} \mathrm{H}_{2} \mathrm{SO}_{4}$ (Certified ACS Plus grade, Fisher Scientific) and 0.5 M KOH (ACS Reagent grade, Sigma Aldrich). Note that the specifications of these reagents allow them to contain transition metal and anionic (e.g., chloride, sulfate, phosphate) impurities in the ppm to tens of ppm range. The electrolytes were used as obtained from the supplier and did not undergo additional purification steps.

To qualitatively assess surface cleanliness and quantitatively estimate the electrochemically active surface area (ECSA), electrolytes were purged with $\mathrm{N}_{2}$ gas (Zero grade, Matheson gas). Cyclic voltammograms were then collected at a fixed negative potential limit of $0 \mathrm{~V}$ vs RHE while the positive potential limit was varied from $0.5 \mathrm{~V}$ to $1.5 \mathrm{~V}$ vs RHE at a scan rate of $100 \mathrm{mV} / \mathrm{s}$.

RHE potential calibration and hydrogen evolution/oxidation catalysis measurements were performed on a rotating disk electrode (RDE) in a sealed Teflon cell (Pine Research). For RDE experiments, a known volume of Pt suspension was dropcast on a $5 \mathrm{~mm}$ diameter glassy carbon electrode for measurements in acid and on $5 \mathrm{~mm}$ Au for analysis in alkaline conditions. The Au substrate in alkaline electrolyte was found to yield improved adhesion, whereas Pt NPs were found to re-suspend when deposited onto glassy carbon. The electrolytes were purged with $\mathrm{H}_{2}$ and the potential was scanned in the range of $-0.15 \mathrm{~V}$ to $+0.1 \mathrm{~V}$ vs RHE in acid and $-0.2 \mathrm{~V}$ to $+0.2 \mathrm{~V}$ vs RHE in base at $10 \mathrm{mV} / \mathrm{s}$. Commercial $10 \mathrm{wt} \% \mathrm{Pt} / \mathrm{C}$ (Alfa Aesar) and a polycrystalline Pt disk electrode (Pine Research) were used as controls.

\section{Results and discussion}

\section{Catalyst synthesis and decapping}

Our synthesis of platinum nanoparticles relies on the reduction of $\mathrm{H}_{2} \mathrm{PtCl}_{6}$ using ascorbic acid and sodium polyacrylate as the capping agent. The synthesis procedure is adopted from prior literature reports, where $\mathrm{Pt}(\mathrm{IV})$ salts have been shown to undergo reduction to metallic $\mathrm{Pt}$ with two equivalents 
of ascorbic acid. $\stackrel{44}{49}$ Sodium polyacrylate polymer as a capping agent offers narrow particle size distribution and has been reported previously for Pt synthesis. $[50-56$ Alkalizing the reaction mixture with sodium hydroxide destabilizes the colloid and facilitates removal of the polyacrylate from the nanoparticle. $\underline{56}$ The main advantage of this approach (over the use of commercial Pt/C catalyst, for example) is that it offers a route to synthesize catalytically active Pt NPs that can be deposited on any substrate and analyzed without the confounding effects of supports or polymer binders. Our synthetic approach also makes use of low-cost reagents (other than the Pt precursor itself) and equipment that is readily available in many wet chemistry laboratories.

We executed TEM, XRD, and FTIR measurements to verify the composition and crystallinity of the Pt NPs before and after removal of the polyacrylate capping agent. Figures $1 \mathrm{1}, \mathrm{b}$ show representative TEM images of capped and uncapped Pt NPs. The particles in panel a clearly remain encapsulated in a matrix of polymer several $\mathrm{nm}$ in diameter. In some cases the polymer encapsulates a single Pt particle and in others several distinct particles are visible, which suggests that the polyacrylate arrests Pt NP growth by encapsulating individual particles, and these core-shell assemblies further aggregate in solution or upon deposition on the TEM grid. By contrast, particles that have been uncapped with alkaline solution aggregate significantly on the TEM grid. Manual analysis of the particle size distribution (see Supporting Information) shows a normal distribution with a mean diameter of $3.1 \pm 0.05 \mathrm{~nm}$ and circularity index of 0.906 . D-spacings extracted from high resolution TEM imaging (insets of Figures 1a,b) further confirm the particle as face-centered cubic (fcc) Pt.

Figures 18,d depict representative XRD and ATR-FTIR data of capped and uncapped Pt NPs. XRD results further confirm fcc Pt as the only crystalline component in the reaction mixture before and after purification. ATR-FTIR data from capped Pt exhibit absorbance features at 1640 $\mathrm{cm}^{-1}$ and $1723 \mathrm{~cm}^{-1}$, which we attribute to carboxylic and/or alcoholic peaks that are blue-shifted relative to the aqueous sodium polyacrylate control. This shift has been previously attributed to the interaction between the particle and the polymer. ${ }^{[6]-62]}$ The uncapped Pt NPs also exhibited absorbance in this range, but at a considerably lower intensity and without clear evidence for a blue 
shift relative to aqueous polyacrylate. This further suggests that the base treament substantially removes polyacrylate from the NPs, but we cannot rule out the possibility that some polymer remains in solution or bound to Pt.
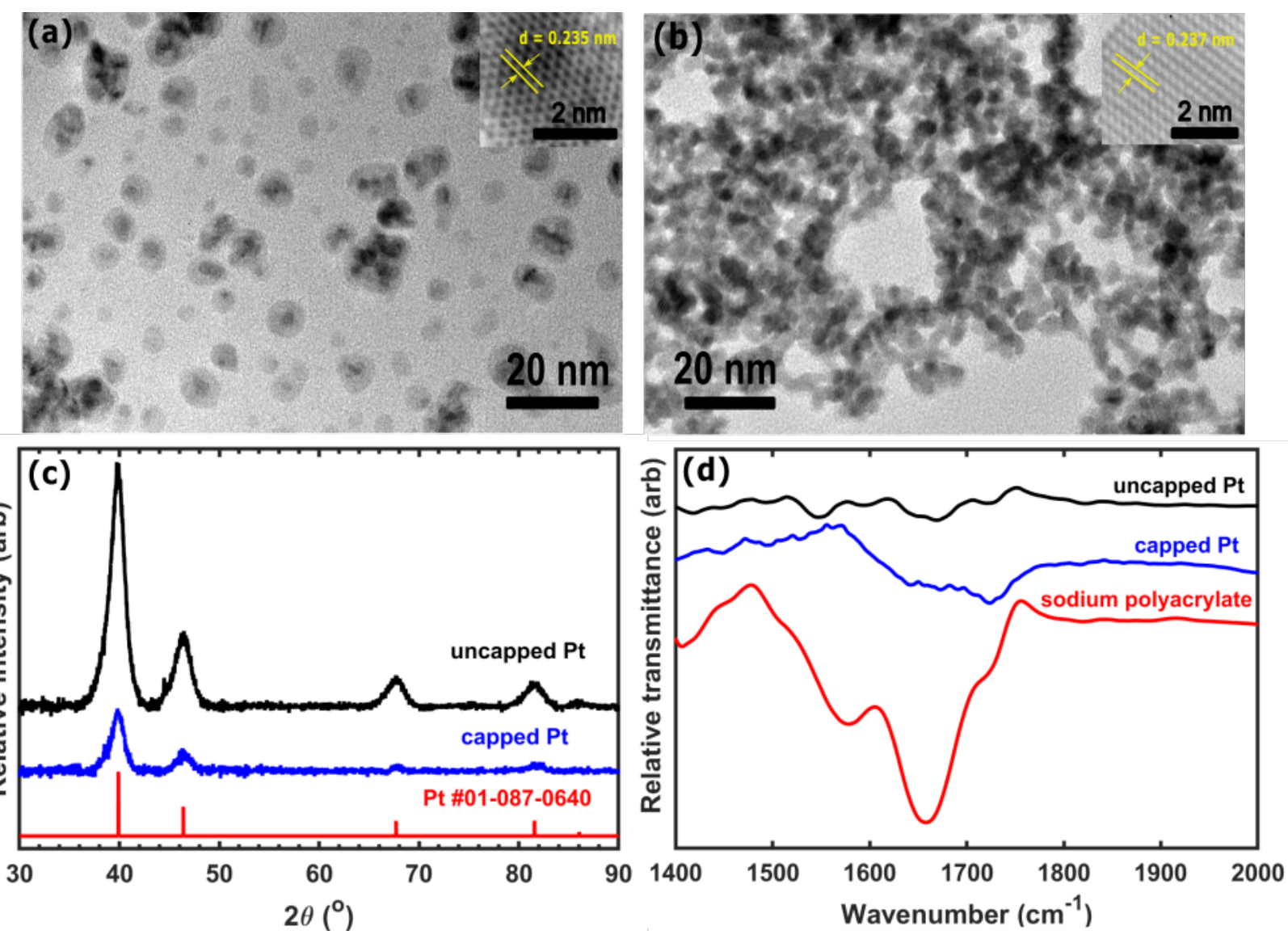

Figure 1: Compiled compositional analysis of Pt nanoparticles via (a,b) TEM, (c) XRD, and (d) ATR-FTIR. The standard XRD pattern of Pt was obtained from the ICDD database via the numerical index indicated in panel (c).

To further characterize the uncapped NPs, we used surface voltammetry to assess the accessibility of Pt surface sites to hydrogen via hydrogen underpotential deposition (HUPD). We evaluated the initial cleanliness of Pt nanoparticles (i.e., the proportion of $\mathrm{Pt}$ surface atoms that are electrochemically accessible) by using HUPD to determine the electrochemically active surface area (ECSA). 19 We also used changes in the ECSA under continuous voltammetric cycling to assess the rate and extent of catalyst deactivation over time. Figure 2 shows representative surface voltammograms of uncapped $\mathrm{Pt}$ nanoparticles in $0.5 \mathrm{M} \mathrm{H}_{2} \mathrm{SO}_{4}$ and in $0.5 \mathrm{M} \mathrm{KOH}$. Using the generally accepted 
HUPD surface charge density of $210 \mu \mathrm{C} / \mathrm{cm}^{2}$, , 63 the ECSA-based specific surface area for uncapped Pt was found to be 16.2 and $12.6 \mathrm{~m}^{2} / \mathrm{g}$ in acid and alkaline electrolytes, respectively. These values can be compared to the expected ECSA of $16.1 \pm 0.3 \mathrm{~m}^{2} / \mathrm{g}$ using the mean particle size obtained from TEM images as $A_{\text {TEM }}=1 / \rho d$ where $\rho$ is the bulk density of Pt and $d$ is the mean particle diameter. Accordingly, despite the tendency for uncapped Pt particles to aggregate-as is clearly evident from TEM imaging - the observed initial ECSA in acid agrees with the value calculated directly from individual particle geometries, implying the particle surface is free of capping agent and other poisons. Moreover, we found similar results for particles deposited from fresh colloids and those that were re-suspended after being allowed to settle, suggesting aggregation does not lead to particle growth at room temperature over timescales of hours to days.

By contrast, the ECSA values extracted from voltammetry in base were consistently lower than those in acid. We tentatively attribute the difference not to residual surface contamination, but instead to a combination of poor adhesion of the unsupported NPs to the electrode surface and a systematic underestimation of the HUPD charge that we attribute to the proximity of the oxygen desorption peak to the HUPD features (see Supporting Information). Nonetheless, these voltammetry results broadly agree with prior literature reports that $\mathrm{NaOH}(\mathrm{aq})$ treatment substantially removes polyacrylate without inducing a significant increase in particle size. ${ }^{43}$ 

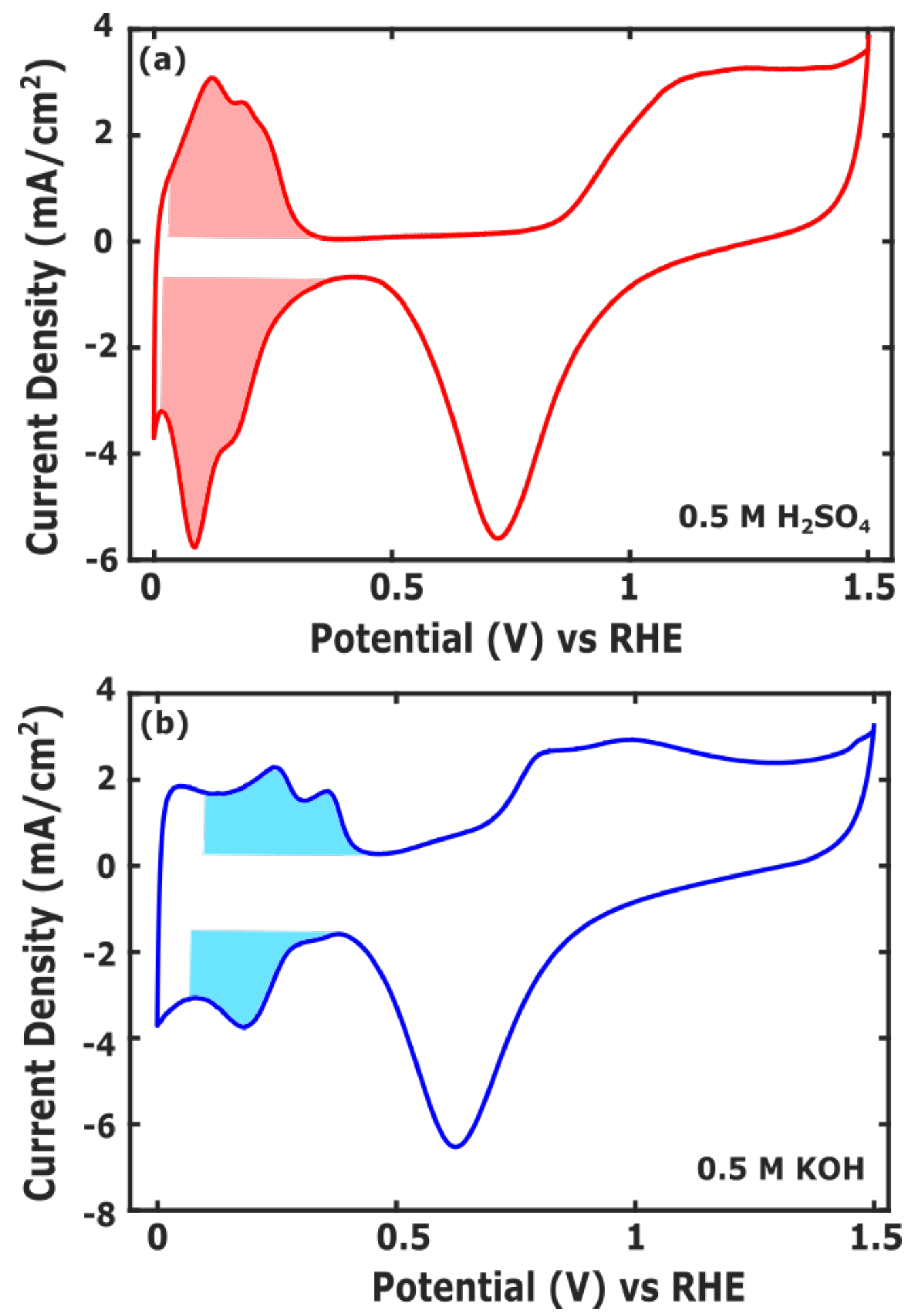

Figure 2: Cyclic voltammogram of Pt nanoparticles in (a) $0.5 \mathrm{M} \mathrm{H}_{2} \mathrm{SO}_{4}$ and (b) $0.5 \mathrm{M} \mathrm{KOH}$ under nitrogen atmosphere at $100 \mathrm{mV} / \mathrm{s}$. The shaded region represents the area used for ECSA calculation. 


\section{Quantifying rates of deactivation in unpurified electrolytes}

Prior literature reports have emphasized the susceptibility of Pt electrocatalysts to deactivation, which negatively impacts activity and lifetime. ${ }^{64} \sqrt[66]{6 e}$ were interested in understanding the mechanism or mechanisms by which our unsupported Pt NPs degrade. To do so, we quantified ECSA decay under continuous voltammetric cycling. Figure 3 presents the results as ECSA versus cycle number in acid and alkaline electrolytes. For this analysis, $0.2 \mathrm{mg} / \mathrm{cm}^{2}$ of Pt NPs were swept at 100 $\mathrm{mV} / \mathrm{s}$ over the potential range from $0 \mathrm{~V}$ to one of three positive potential limits: $0.5,1.0$, and 1.5 $\mathrm{V}$ vs. RHE for a total of 50 cycles. A different Pt film was used for each potential window and 15 such films obtained from 5 different synthesis batches were tested for the analysis.

In acid, the initial ECSA showed a pronounced increase with increasing positive potential, which suggests that the cleaning effect of the first cycle involves oxidative removal of contaminants. A monotonic decrease in ECSA was observed in all cases over 50 cycles. Moreover, we observed that the relative (normalized to initial ECSA) rate of ECSA loss per cycle was nearly identical when Pt nanoparticles were scanned at $0.5 \mathrm{~V}$ and $1 \mathrm{~V}$ vs RHE, suggesting similarity in their deactivation mechanisms. When scanned to $1.5 \mathrm{~V}$ vs RHE, the rate of deactivation was initially lower, but accelerated after several cycles such that the total fractional loss in surface area was $\sim 40 \%$ in all cases.

ECSA values also decreased monotonically with cycle number in alkaline electrolyte across all positive potential limits. However, the relationship between initial ECSA and potential limit was reversed relative to the acid case, with the least positive potential limit resulting in the highest initial ECSA. We believe this difference again is attributable to the proximity of the oxygen desorption feature to the HUPD region, which results in a systematic underestimate of the ECSA when significant surface coverages of $\mathrm{PtO}_{\mathrm{x}}$ are generated in the positive-going sweep (see Supporting Information). The relative rates of deactivation in base were also found to be similar at $1 \mathrm{~V}$ and $1.5 \mathrm{~V}$ vs RHE, whereas the less positive potential limit resulted in marginally slower ECSA loss. These observations are consistent with the ability of aqueous base to remove residual capping agent from the $\mathrm{Pt}$ NP surface (which negates the benefit of positive potential cycling) and suggests the presence of a 
second deactivation mechanism that dominates at positive potentials.
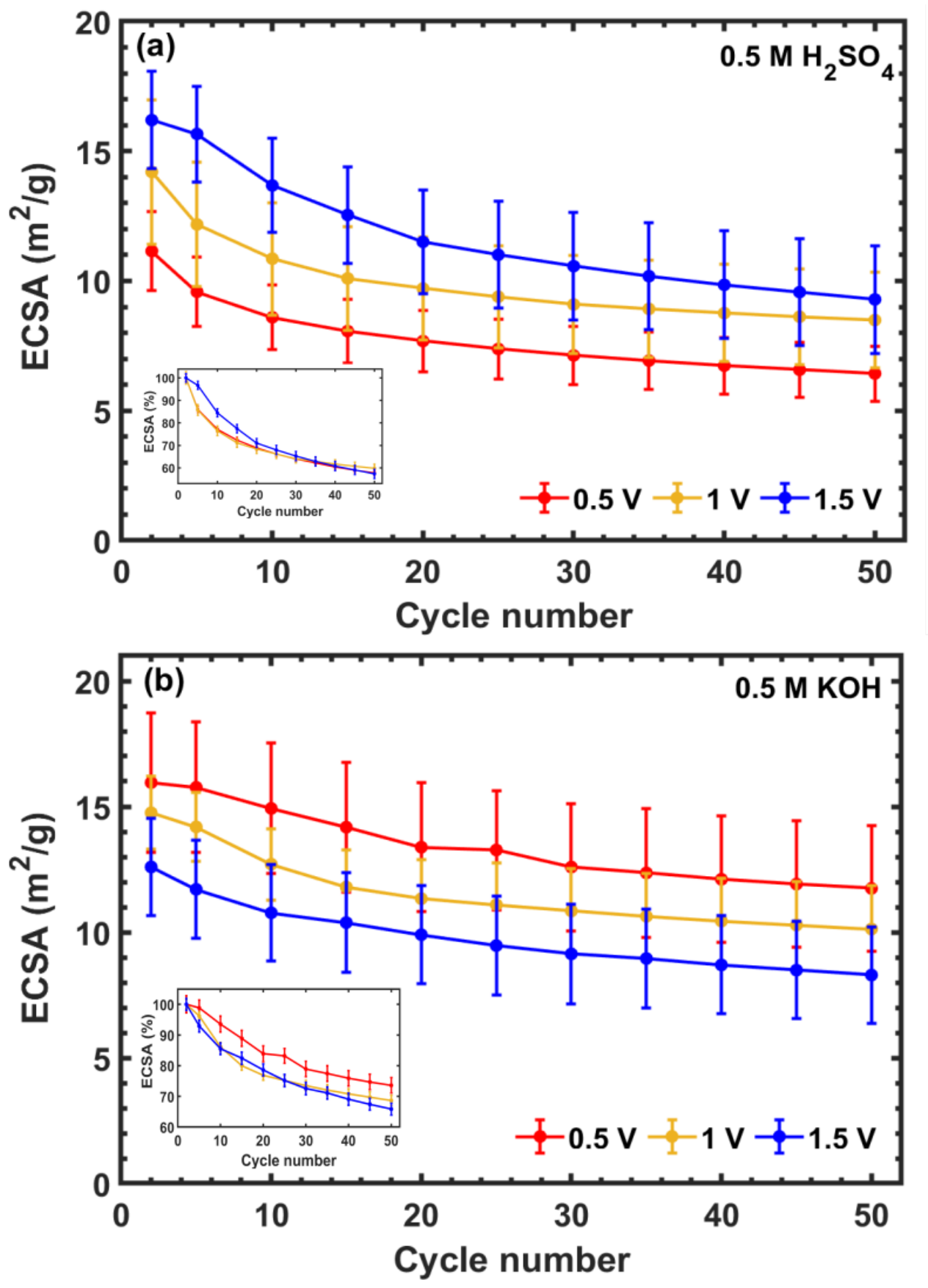

Figure 3: Variation of Pt NP ECSA as a function of cycle number when cycled between a fixed negative scan limit of $0 \mathrm{~V}$ vs RHE and the noted positive scan limits over 50 cycles in (a) $0.5 \mathrm{M}$ $\mathrm{H}_{2} \mathrm{SO}_{4}$ and (b) $0.5 \mathrm{M} \mathrm{KOH}$. Experimental conditions were otherwise identical to that in Figure 2. Error bars represent $95 \%$ confidence intervals from 15 distinct films. The inset shows the relate rate of ECSA decay versus cycle number by normalizing to that of the first full cycle.

We consider it significant that these Pt NPs lost 25-40 \% of their initial ECSA over just 50 
voltammetric cycles, which required as little as $\sim 10$ minutes. This extent of ECSA loss is large enough to conclude that the Pt particles are no longer pristine, thereby calling into question their use as experimental controls or activity benchmarks. Hence, it is reasonable to adopt as a general guideline that unsupported Pt NPs of this type should be freshly deposited onto a clean electrode at relatively short time intervals (every few minutes or perhaps between each individual measurement) if they are to be used to good effect in unpurified electrolytes.

\section{Differentiating between several modes of deactivation}

Several deactivation mechanisms are understood to proceed for Pt catalysts under electrochemical conditions. These include catalyst poisoning via surface adsorption or electrodeposition of impurities; oxidative dissolution, which could be chemically or electrochemically induced; particle growth resulting from Ostwald ripening or similar processes; and particle detachment from the electrode substrate. In acidic electrolyte, unsupported Pt nanoparticles are known to undergo dissolution by

repeated cycling across the potential range required to generate and reduce Pt-oxides. ${ }^{65}[69$ The dissolution proceeds primarily during the reductive scan when platinum oxide is nominally reduced back to metallic $\mathrm{Pt}$; this reaction proceeds in parallel with dissolution of $\mathrm{Pt}^{2+}$ ions. Under continuous cycling, these ions may remain in the electrolyte or they may be redeposited as the electrode potential is swept further negative. This dissolution-redeposition process may then induce particle growth via electrochemically accelerated Ostwald ripening. ${ }^{70171}$ Nevertheless, it is difficult to entirely eliminate oxidative cycling in practice, as this treatment is also highly effective at removing organic and/or inorganic contaminants from the Pt surface, either through catalytic oxidation or as a direct result of the dissolution of Pt surface layers. ${ }^{72}$

The deactivation of Pt nanoparticles has not been as thoroughly characterized in alkaline conditions as in acid. Deposition of transition metal impurities on the electrode surface and analogous dissolution processes have each been implicated as the root causes of reduced catalytic activity. 20 Our ECSA variation versus cycle number data in figure 3 yield puzzling results where the initial ECSA values and the potential window are not consistent across acid and base conditions. More- 
over, the relative rates of deactivation (20-40\% over 50 cycles) are similar across nearly all conditions studied. These observations leave open the question of whether the dominant mechanism of ECSA loss is similar or different across all potential windows and $\mathrm{pH}$ conditions.

Figure 4 compiles the results of an experiment directed at differentiating between reversible catalyst poisoning and irreversible loss of surface area, wherein Pt NP films were first subjected to 50 cycles to a positive limit of $0.5 \mathrm{~V}$ vs RHE, followed by 50 cycles to $1.5 \mathrm{~V}$ vs RHE, and then a third set of 50 cycles to $0.5 \mathrm{~V}$. The ECSA decay on scanning at $0.5 \mathrm{~V}$ vs RHE for the first 50 cycles in acid and alkaline solutions again resulted in a 20-40\% loss in ECSA. When the positive potential was shifted to $1.5 \mathrm{~V}$ vs RHE, ECSA values in acid recovered over $\sim 5$ cycles and in some cases even exceeded the initial values. This indicates that the Pt surface was renewed after scanning at oxidizing potential, which strongly suggests catalyst poisoning dominates at $0.5 \mathrm{~V}$ vs RHE. Nonetheless, after the initial recovery at $1.5 \mathrm{~V}$ vs RHE, the ECSA decayed again by nearly the same amount as in the initial set of 50 cycles. Returning the potential window to a $0.5 \mathrm{~V}$ positive limit had little further impact on the ECSA, which continued to diminish slowly through a final set of 50 cycles. These data suggest the mechanism by which ECSA diminishes when cycling to a more positive potential limit is irreversible_-dissolution, particle growth, or detachment.

By contrast to the results in acid, when cycled in alkaline electrolyte, the ECSA did not recover when the voltage range was modulated from 0.5 to $1.5 \mathrm{~V}$ and back to $0.5 \mathrm{~V}$. Instead we observed a nearly continuous decrease in surface area over 150 cycles, with a systematic offset for data collected to $1.5 \mathrm{~V}$ vs RHE again attributable to systematic errors resulting from the Pt surface oxide reduction feature in the voltammogram.

Our observations in acid agree with prior reports that electrochemical oxidation is highly effective at removing catalyst poisons from Pt in acid. ${ }^{72]}$ Many organic (e.g., hydrocarbons) and inorganic (e.g., sulfate, chloride) species from the synthesis mixture, the electrolyte, or the atmosphere may adsorb to the Pt surface and can be removed by cycling to positive potentials. ${ }^{[2+74}$ By contrast, oxidative electrochemical cleaning did not renew the catalyst surface in base. There are at least three possible explanations for this observation. First, the catalyst surface may become contaminated 

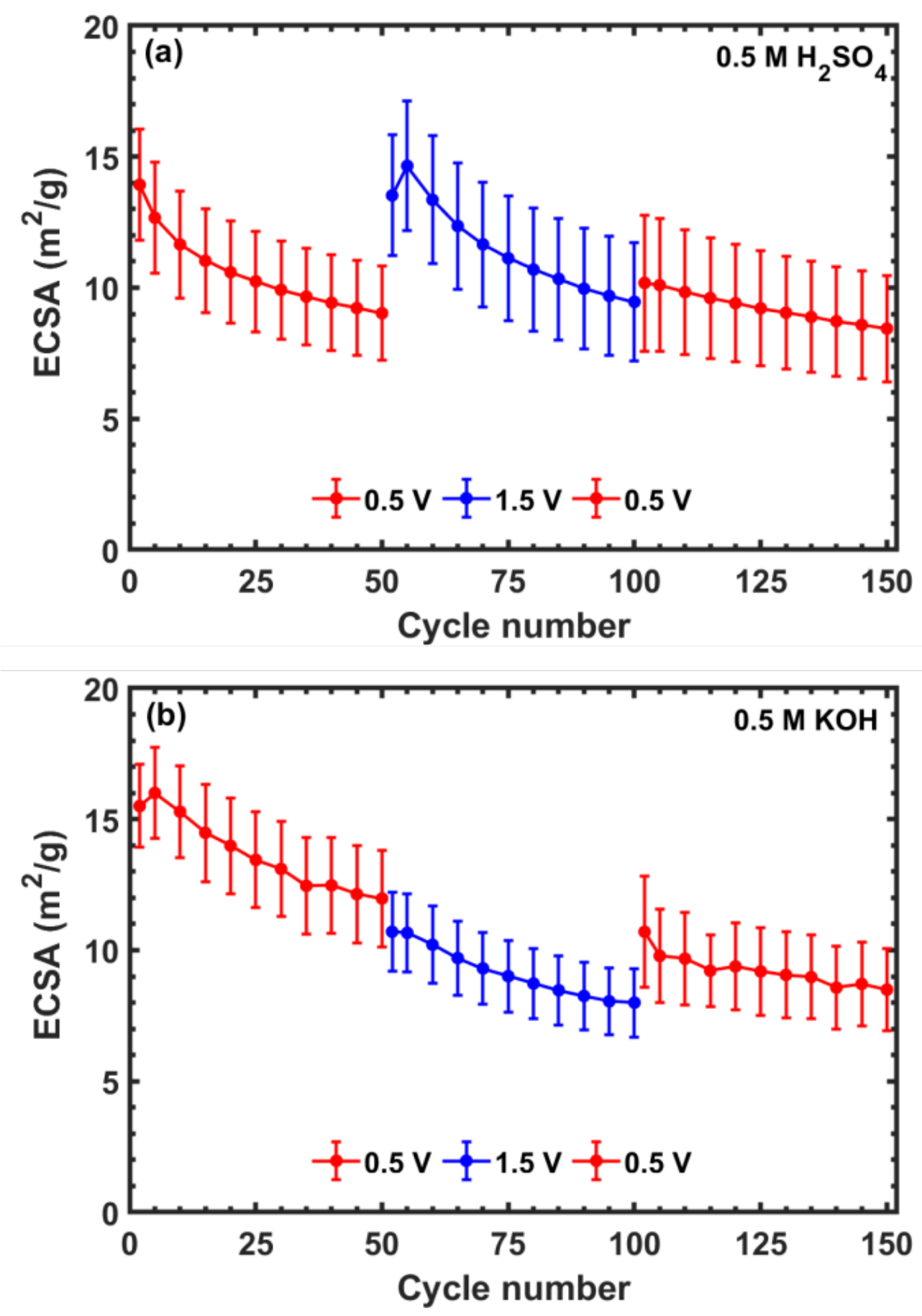

Figure 4: ECSA versus cycle number for Pt NP films scanned to narrow potential of $0.5 \mathrm{~V}$ for 50 cycles, followed by positive potential limit of $1.5 \mathrm{~V}$ for 50 cycles and again followed by $0.5 \mathrm{~V}$ for 50 cycles in (a) $0.5 \mathrm{M} \mathrm{H}_{2} \mathrm{SO}_{4}$ and (b) $0.5 \mathrm{M} \mathrm{KOH}$. Experimental conditions and error bounds were otherwise identical to those in Figures 2 and 3. 
with a non-oxidizable poison over all potential windows. A second explanation is that alkaline conditions facilitate detachment of nanoparticles from the electrode surface, and this may proceed at some relatively constant rate regardless of cycling conditions. A third explanation is that the Pt NPs undergo continuous particle growth in alkaline conditions under potential cycling that again depends weakly, if at all, on cycling conditions.

To further differentiate between loss of surface area via particle growth, dissolution, and detachment, we performed identical location transmission electron microscopy (IL-TEM) experiments on catalyst thin films before and after they were subjected to CV cycling over a range of potential windows for 50 cycles at $100 \mathrm{mV} / \mathrm{s}$. Figure 5 compiles the results of these experiments in acid electrolyte. At $0.5 \mathrm{~V}$ vs RHE, the TEM images before and after cycling do not show significant changes in the particle morphology. The overall particle size distribution also did not significantly change before and after cycling. Similar results were observed when Pt NPs were scanned to $1 \mathrm{~V}$ vs RHE in acid. However, a stark difference was observed when the particles were cycled to $1.5 \mathrm{~V}$ vs RHE, where the TEM images clearly show particle growth. The particle size distribution data confirmed that the mean particle diameter increases by slightly more than two-fold. A similar analysis was also performed in alkaline solution, where scanning at $0.5 \mathrm{~V}$ and $1 \mathrm{~V}$ vs RHE did not result in a significant increase in particle size but cycling at $1.5 \mathrm{~V}$ vs RHE resulted in a $\sim 30 \%$ increase in mean particle diameter (see Supporting Info). 


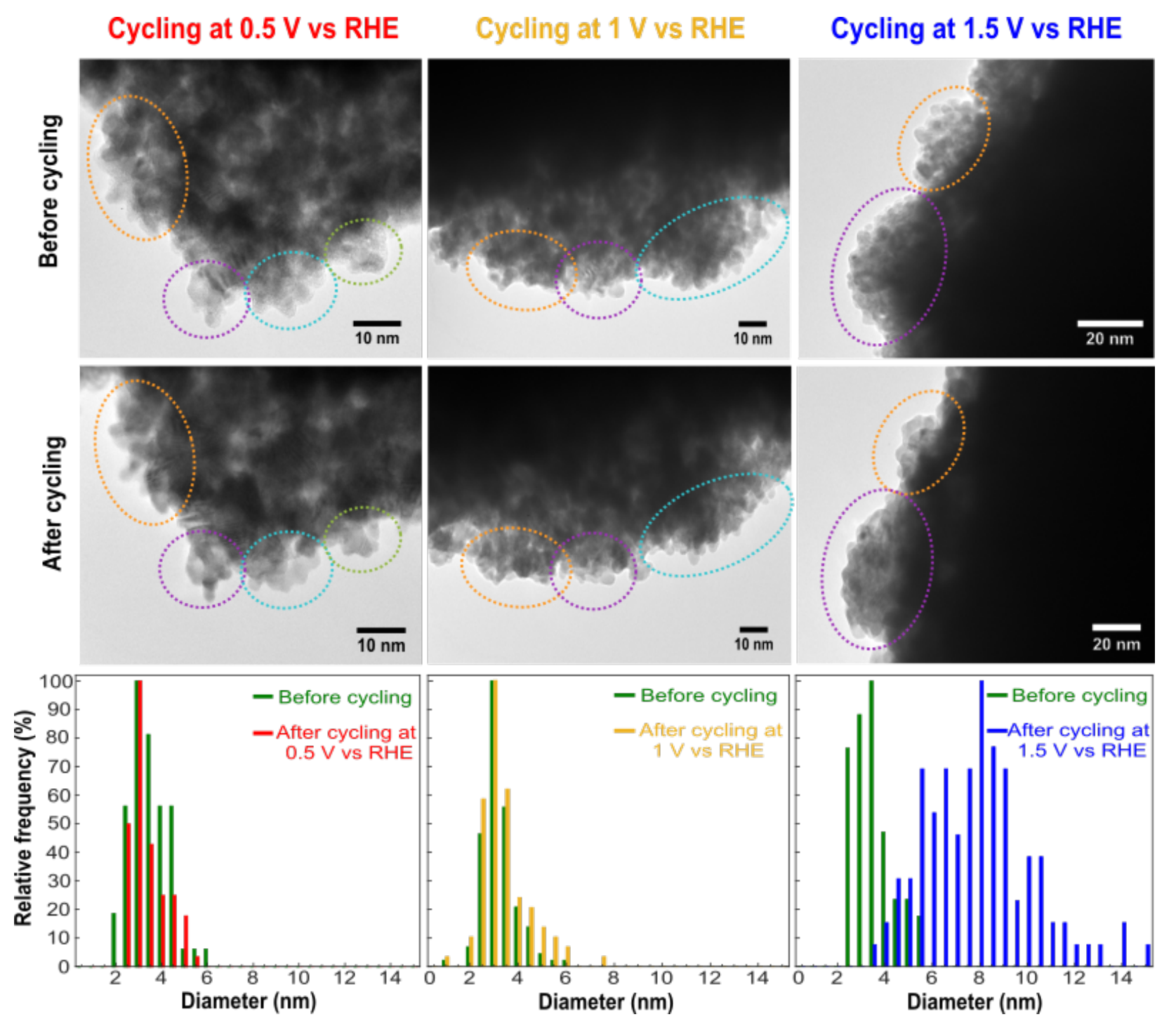

Figure 5: Identical location TEM images and particle size distributions of Pt nanoparticles before and after cycling at three different positive limit potentials in $0.5 \mathrm{M} \mathrm{H}_{2} \mathrm{SO}_{4}$.

Combining the ECSA and IL-TEM analysis, it is evident that when the potential is cycled at least as positive as $1 \mathrm{~V}$ vs RHE, catalyst poisoning is the dominant mechanism by which ECSA diminishes. This is evidenced by the fact that we saw no changes in particle size before and after cycling. But when samples were cycled to $1.5 \mathrm{~V}$ vs RHE, the particle size increased significantly in acid and base. We therefore attribute the growth of Pt nanoparticles to Ostwald ripening, which may be facilitated by transient Pt dissolution and redeposition during cycling. ${ }^{33}$ The observed decrease in ECSA in base at $1.5 \mathrm{~V}$ vs RHE can be fully attributed to the increase in particle size. By contrast, 
the observed loss in ECSA in acid was smaller than that predicted from increase in particle size. We speculate that this discrepancy may result from a modest increase in atomic scale roughness (i.e., increased surface disorder) for Pt NPs in acid that also results from continuous formation and reduction of Pt-oxides.

It is striking that, despite evidence that at least two different potential and $\mathrm{pH}$-dependent mechanisms are involved, the observed rate of Pt NP deactivation was remarkably consistent — tens of percent loss in ECSA over tens of minutes-across all conditions studied. Absent evidence to the contrary, we conclude that this consistent rate of ECSA loss is coincidental, and in fact the comparative rates of catalyst poisoning and particle growth would likely be different in more highly purified or severely contaminated electrolytes. Indeed, prior work on Pt NPs under scrupulously clean conditions showed that stable cycling can be achieved when the potential is maintained negative of $1 \mathrm{~V}$ vs RHE. ${ }^{4354}$ However, it is challenging and costly to obtain this level of cleanliness; hence, it may be preferable in many cases to simply refresh the Pt NP film by removing "spent" particles from the electrode substrate and replacing with a fresh NP film. This approach is directly analogous to the ubiquitous use of abrasive polishing to clean polycrystalline Pt electrodes between experiments, with the added benefit of the ability to control the Pt loading, and hence the areal density of active sites.

\section{The effect of Pt deactivation on benchmark measurements}

Upon determination that our Pt NPs initially exhibit high accessibility to adsorbed hydrogen, we endeavored to assess their ability to perform routine measurements associated with HER/HOR catalyst benchmarking. Specifically, we sought to identify experimental timescales over which our Pt NPs exhibit reduced catalytic activity toward hydrogen evolution/oxidation in reagent-grade electrolytes.

Figure 6 shows representative HER/HOR polarization curves of Pt nanoparticles in comparison with a commercial Pt/C catalyst at Pt mass loading of $0.2 \mathrm{mg} / \mathrm{cm}^{2}$. Note that, based on ECSA measurements and known particle sizes, these electrodes exhibit roughness factors (electractive 
surface area normalized to project area of the electrode) of $\sim 30$. We also included a simulated concentration overpotential curve (simulation details can be found in the Supporting Information), which represents the current-overpotential response of an ideal nonpolarizable electrode whose activity is limited only by mass transfer. In acid, the HER activity of Pt/C and our unsupported Pt NPs are nominally identical and overlay with the concentration overpotential curve. This means that the hydrogen evolution/oxidation reaction on Pt is purely transport limited, and the true kinetics of the catalyst are not directly measurable. We then cycled the unsupported Pt NPs in the HER/HOR region for $\sim 30$ minutes from -0.15 to $+0.1 \mathrm{~V}$ vs RHE at $100 \mathrm{mV} / \mathrm{s}$ and measured the HER/HOR performance again. No change was observed in the current-overpotential data after cycling, which suggests that any loss in catalytic activity was not sufficient to induce a kinetic limitation.

This result illustrates several important properties of Pt catalysts when used for hydrogen evolution/oxidation measurements in acid. First, a clean, high surface area Pt catalyst film should generally yield transport-limited activity for the HER/HOR in aqueous acid unless steps are taken to greatly increase the mass transfer coefficient beyond what is achievable using conventional techniques like RDE voltammetry. $\frac{75176}{17}$ This means the activity should be indistinguishable from that of a catalyst with a heterogeneous electron transfer of infinity. Such a nanostructured Pt catalyst will continue to exhibit transport-limited activity even after it becomes poisoned or otherwise deactivated, provided the remaining areal site density remains sufficiently high. A recent report showed that areal loadings of unsupported $3.8 \mathrm{~nm}$ Pt NPs needed to be reduced to $<1 \mu \mathrm{g} / \mathrm{cm}^{2}$ to observe kinetically limited behavior. ${ }^{22}$ This would be roughly equivalent to a $>99.5 \%$ loss in ECSA at the $0.2 \mathrm{mg} / \mathrm{cm}^{2}$ loading we used here. Accordingly, unsupported Pt NPs and commercial Pt/C catalysts can certainly be used to benchmark the activity of a new HER/HOR catalyst, even in unpurified reagent-grade electrolyte. However, if a novel catalyst is found to exhibit higher activity toward the HER or HOR than a Pt control in acid solution, it is in fact very unlikely that the catalyst of interest is "better than Pt," but rather the Pt control has likely been severely contaminated by electrolyte impurities or another source.

We also performed HER/HOR measurements in alkaline electrolyte, which gave considerably 
different results. The activity of $\mathrm{Pt} / \mathrm{C}$ and $\mathrm{Pt}$ nanoparticles was similar, and both were lower than the simulated concentration overpotential. This is consistent with numerous prior reports showing that $\mathrm{Pt}$ is less active toward hydrogen evolution/oxidation under alkaline conditions than in acid. 1976 Cycling of Pt nanoparticles in the HER region from $-0.2 \mathrm{~V}$ to $+0.2 \mathrm{~V}$ vs RHE for $\sim 30$ minutes also resulted in $\sim 30 \%$ reduction in current density. This agrees well with our ECSA data showing that accessible Pt surface sites decreased by tens of \% over tens of minutes of continuous cycling. It is also consistent with the expectation that Pt HER/HOR electrocatalysis is kinetically limited in base, even for electrodes with relatively high Pt surface areas; hence, catalyst deactivation manifests as a proportional decrease in activity. These results collective suggest that Pt-based control measurements in alkaline electrolytes are considerably more sensitive to the cleanliness and sample history than those in acid. Comparisons between Pt and novel HER/HOR catalysts in alkaline conditions are also more relevant, since Pt remains limited by reaction kinetics over a wide range of catalyst loadings and it is indeed possible to obtain catalytic activities exceeding that of pure Pt. 77.79

As a final assessment of the utility of our Pt NPs for catalyst benchmarking, we used opencircuit potential (OCP) measurements in hydrogen-purged electrolytes to calibrate our $\mathrm{Ag} / \mathrm{AgCl}$ reference electrode to the reversible hydrogen electrode (RHE) potential. This procedure is crucial in characterizing catalysts for reactions involving proton-electron transfer because it enables accurate determination of catalyst overpotentials relative to an empirically measured, $\mathrm{pH}$-dependent thermodynamic equilibrium potential. Figure 7 depicts representative RHE calibrations using our Pt NPs alongside polycrystalline Pt disk electrodes in acid and base. For consistency, these measurements were initiated in electrolytes that were first purged with atmospheric air for several minutes ( $\sim 1000$ seconds) until a stable potential was attained. The purge gas was then switched to hydrogen at the same flow rate. The open-circuit potential was found to decrease over several minutes as dissolved oxygen was replaced with hydrogen until it asymptotically approached a stable potential in the expected range for a reversible hydrogen electrode (near $-0.25 \mathrm{~V}$ vs. $\mathrm{Ag} / \mathrm{AgCl}$ in $0.5 \mathrm{M} \mathrm{H}_{2} \mathrm{SO}_{4}$ and $-1.0 \mathrm{~V}$ in $0.5 \mathrm{M} \mathrm{KOH}$ ). Figures $7 \mathrm{k}$ and d show detailed views of a narrow range of potentials in the asymptotic region. In acid, OCP values for the Pt disk electrode and unsupported Pt nanopar- 

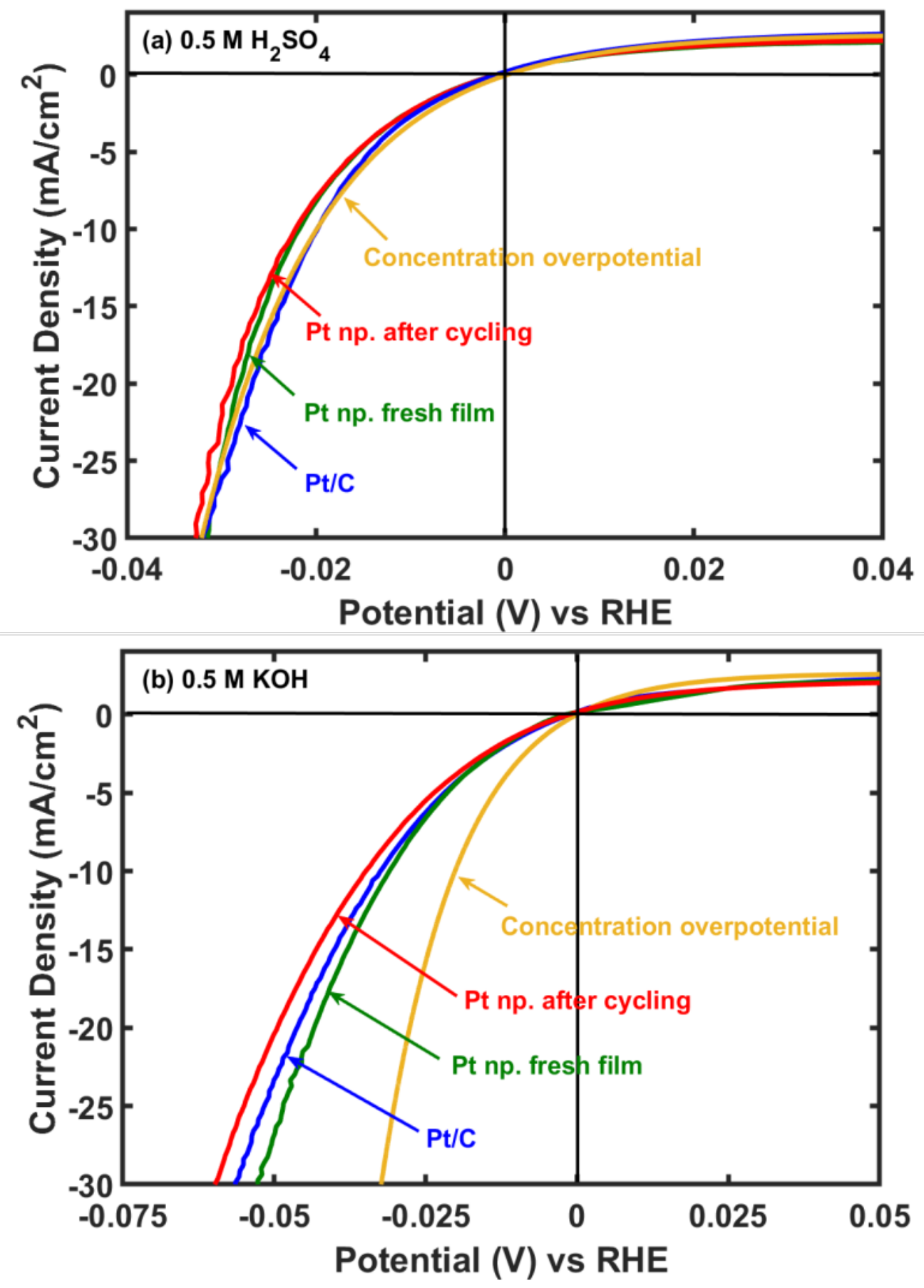

Figure 6: HER/HOR polarization curve of fresh film of Pt nanoparticles and after cycling in the HER potential range for 30 minutes (i.e.at $100 \mathrm{mV} / \mathrm{s}$ for 300 cycles)in comparison with commercial $10 \mathrm{wt} \% \mathrm{Pt} / \mathrm{C}$ and simulated diffusion overpotential curve under hydrogen gas purge at $1600 \mathrm{rpm}$ in (a) $0.5 \mathrm{M} \mathrm{H}_{2} \mathrm{SO}_{4}$ and (b) in $0.5 \mathrm{M} \mathrm{KOH}$. The Pt mass loading was kept constant at $0.2 \mathrm{mg} / \mathrm{cm}^{2}$. 
ticles remained stable within $\pm 2 \mathrm{mV}$ over the last 10 minutes of the measurement. The minimum OCP values, which can be interpreted as an estimate of $0 \mathrm{~V}$ vs RHE, were -0.266 and $-0.270 \mathrm{~V}$ vs $\mathrm{Ag} / \mathrm{AgCl}$; the modest difference between these values constitutes uncertainty in the RHE calibration attributable to, e.g., reference electrode drift or incomplete exclusion of atmospheric air during the hydrogen purge. These data show that clean Pt electrodes of varying surface roughness remain sufficiently catalytic over the measurement interval to obtain a stable equilibrium between hydrogen and protons.

In alkaline solution, Pt nanoparticles gave a similarly stable final OCP value, but the Pt disk was found to drift positive by $\sim 30 \mathrm{mV}$ over several minutes. This indicates that this relatively low surface-area electrode becomes sufficiently deactivated on the timescale of the RHE calibration to exhibit a mixed potential likely involving equilibration to the HER/HOR equilibrium and one or more additional equilibria associated with impurities in solution or adsorbed on the electrode. 80 These data again exemplify the greater sensitivity of Pt to deactivation in base, which results from its lower catalytic activity toward hydrogen evolution/oxidation and can be ameliorated by increasing the areal density of Pt surface sites. 

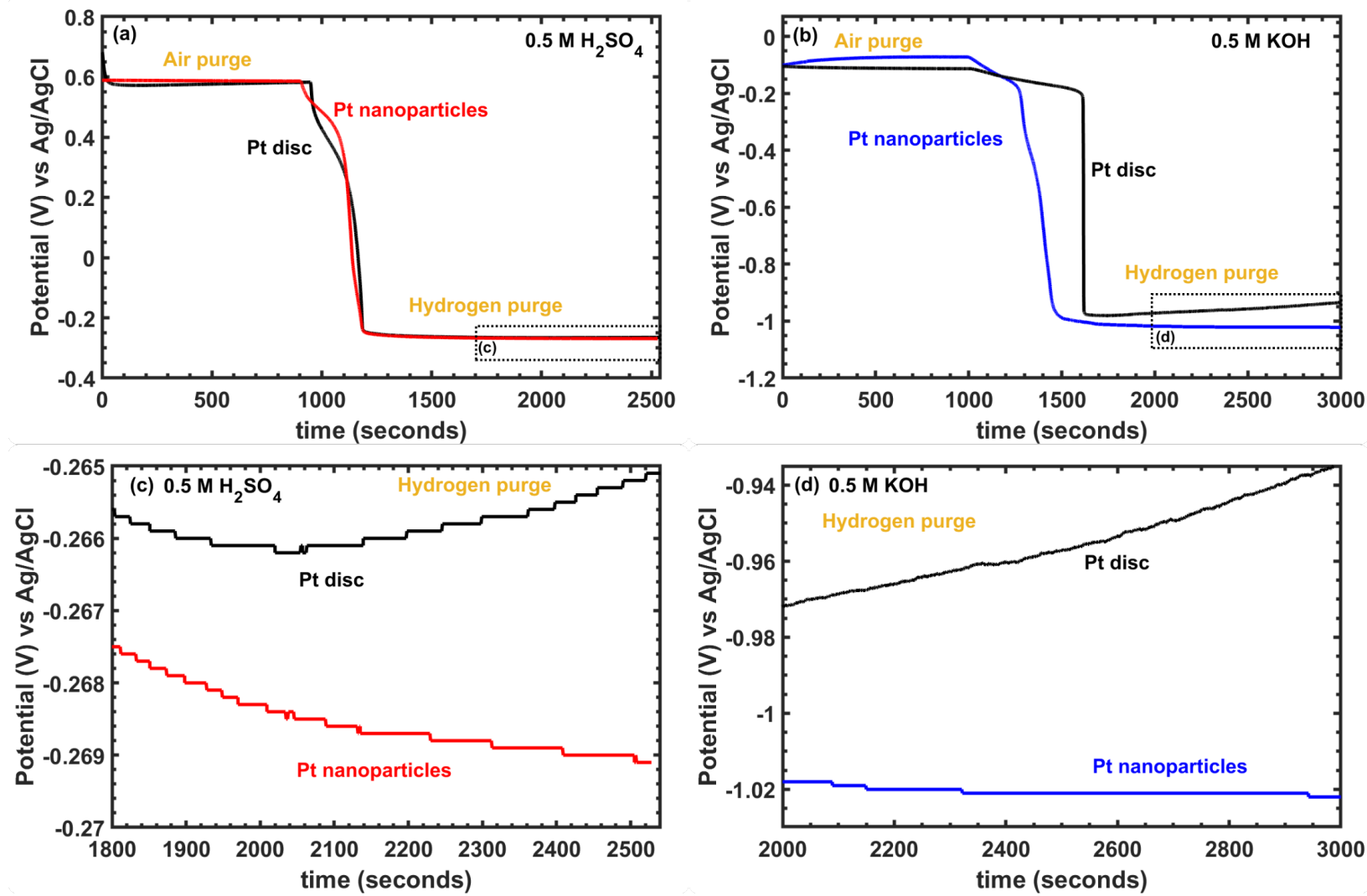

Figure 7: Open circuit potential measurements of Pt nanoparticles and Pt disk electrodes under air purge followed by $\mathrm{H}_{2}$ purge in (a) $0.5 \mathrm{M} \mathrm{H}_{2} \mathrm{SO}_{4}$ and (b) $0.5 \mathrm{M} \mathrm{KOH}$. Panels (c) and (d) are details of the potential vs time data in (a) and (b), respectively, over a narrower range of potentials after the solution became saturated with $\mathrm{H}_{2}$.

\section{Conclusions}

In summary, we synthesized uniform, $3 \mathrm{~nm}$ unsupported Pt nanoparticles via a straightforward aqueous route involving ascorbic acid and polyacrylate as reductant and capping agent, respectively. The resulting Pt NP colloidal suspension can be purified by simply basifying the reaction mixture, which substantially removes the polyacrylate capping agent. The purified particles remain stably suspended in pure water, which lends well to their use as a laboratory standard for electrochemical hydrogen evolution/oxidation and potentially in numerous other applications. We further showed that these catalyst particles lose considerable active surface area over a span of tens of minutes during continuous electrochemical cycling in unpurified, reagent grade aqueous acid and 
base. We contextualized these results in terms of the utility of these Pt NPs for benchmarking novel HER/HOR catalysts. We further demonstrated that Pt deactivation has a much greater negative impact on these measurements in base than in acid.

Considering together all the results reported here, we conclude that it is indeed possible to collect accurate Pt HER/HOR activity benchmark data and to calibrate reference electrodes relative to the RHE without the need to rigorously purify electrolytes or otherwise maintain scrupulously clean conditions. To do so consistently, we recommend adopting the following practices:

1. When possible, maximize the areal density of Pt active sites by using Pt NPs with mass loading $\geq 0.1 \mathrm{mg} / \mathrm{cm}^{2}$ or platinized Pt electrodes.

2. When using Pt NPs, compare the results of ECSA measurements against the surface area expected from independently measured particle size/shape to determine whether they are free of surface site-blocking impurities.

3. When operating in an acidic environment, Pt catalysts may be further cleaned by scanning to oxidizing potentials on the order of $1.5 \mathrm{~V}$ vs RHE. However, these treatments should be limited to a short duration (no more than a few minutes), after which particle films should simply be removed from the electrode substrate and a fresh film deposited.

4. Do not use potential cycling to clean Pt catalysts in alkaline electrolytes, as is has no beneficial effect. Instead, measurements should be limited to a few tens of minutes or less, between which catalyst films should be removed and refreshed. Experiments that require longer durations will likely require electrolyte purification.

5. Regularly execute internal checks for catalyst deactivation. These include hydrogen evolution/oxidation measurements in acid, which should give mass-transfer limited activity, and RHE calibrations, which should yield little to no potential drift over at least several minutes. 


\section{Acknowledgement}

We acknowledge the Arnold and Mabel Beckman Foundation and the Mascaro Center for Sustainable Innovation for financial support of this work. The TEM work was performed at the Nanoscale Fabrication and Characterization Facility, a laboratory of the Gertrude E. and John M. Petersen Institute of NanoScience and Engineering, housed at the University of Pittsburgh. We also acknowledge Prof. Leanne Gilbertson for the use of an ultramicrobalance.

\section{Supporting Information Available}

Synthesis schematics; Cyclic voltammograms of capped and uncapped Pt nanoparticles; Additional ECSA analysis; Diffusion overpotential simulation details;ECSA and IL-TEM analysis of Pt nanoparticles deposited on TEM grids.

\section{References}

(1) Chaston, J. The growing industrial use of the platinum metals. Platinum Metals Review 1982, 26, 3-9.

(2) Matthey, J. About PGM - PMM. 2015, 2-3.

(3) Bell, T. The Properties and Applications of Platinum. 2020.

(4) World Platinum Investment Council, Platinum Industrial Demand. 2020; https://www. cmegroup.com/education/articles-and-reports/platinum-industrial-demand . html? source=rss,

(5) Shaffer, C. Platinum Group Metals at Work : Industrial Applications Chemical processing Nitric acid. Accelerating Science 2015, 1-4. 
(6) Twigg, M. V. Progress and future challenges in controlling automotive exhaust gas emissions. Applied Catalysis B: Environmental 2007, 70, 2-15.

(7) Paraskevas, M. S.; Papoutsi, K.; Ochsenkühn-Petropoulou, M. A Novel Way of Sampling Particles Containing Platinum Group Elements from Automobile Catalysts. Analytical Letters 2012, 45, 539-550.

(8) Moldovan, M.; Gömez, M. M.; Palacios, M. A. Determination of platinum, rhodium and palladium in car exhaust fumes. Journal of analytical atomic spectrometry 1999, 14, 11631169.

(9) Golunski, S. E. Why use platinum in catalytic converters? Platinum Metals Review 2007, 51, 162.

(10) Salman, A. u. R.; Enger, B. C.; Auvray, X.; Lødeng, R.; Menon, M.; Waller, D.; Rønning, M. Catalytic oxidation of NO to NO2 for nitric acid production over a Pt/A12O3 catalyst. Applied Catalysis A: General 2018, 564, 142-146.

(11) Grande, C. A.; Andreassen, K. A.; Cavka, J. H.; Waller, D.; Lorentsen, O. A.; Øien, H.; Zander, H. J.; Poulston, S.; García, S.; Modeshia, D. Process Intensification in Nitric Acid Plants by Catalytic Oxidation of Nitric Oxide. Industrial and Engineering Chemistry Research 2018, 57, 10180-10186.

(12) Lewis, L. N.; Stein, J.; Gao, Y.; Colborn, R. E.; Hutchins, G. Platinum catalysts used in the silicone industry. Platinum Matals Reviews 1997, 41, 66-75.

(13) Lukin, R. Y.; Kuchkaev, A. M.; Sukhov, A. V.; Bekmukhamedov, G. E.; Yakhvarov, D. G. Platinum-catalyzed hydrosilylation in polymer chemistry. Polymers 2020, 12, 1-22.

(14) Pellegrino, J.; Brueske, S.; Carole, T.; Richlen, S.; Valentine, B. Energy and Environmental Profile of the U.S. Petroleum Refining Industry; 2007; pp 68-70. 
(15) Kapustin, V.; Chernysheva, E.; Maximova, A.; Zinchenko, Y. Development of new catalytic processes for processing petroleum feedstock. Pure and Applied Chemistry 2017, 89, 1579_ 1585 .

(16) Alia, S. M.; Pivovar, B. S. Evaluating Hydrogen Evolution and Oxidation in Alkaline Media to Establish Baselines. Journal of The Electrochemical Society 2018, 165, F441-F455.

(17) Zheng, J.; Sheng, W.; Zhuang, Z.; Xu, B.; Yan, Y. Universal dependence of hydrogen oxidation and evolution reaction activity of platinum-group metals on $\mathrm{pH}$ and hydrogen binding energy. Science Advances 2016, 2, 1-9.

(18) Zadick, A.; Dubau, L.; Sergent, N.; Berthomé, G.; Chatenet, M. Huge Instability of Pt/C Catalysts in Alkaline Medium. ACS Catalysis 2015, 5, 4819-4824.

(19) Sheng, W.; Gasteiger, H. A.; Shao-Horn, Y. Hydrogen Oxidation and Evolution Reaction Kinetics on Platinum: Acid vs Alkaline Electrolytes. Journal of The Electrochemical Society 2010, 157, B1529.

(20) Weber, D. J.; Dosche, C.; Oezaslan, M. Fundamental Aspects of Contamination during the Hydrogen Evolution/Oxidation Reaction in Alkaline Media. Journal of The Electrochemical Society 2020, 167, 024506.

(21) Weber, D. J.; Janssen, M.; Oezaslan, M. Effect of Monovalent Cations on the HOR/HER Activity for Pt in Alkaline Environment. Journal of The Electrochemical Society 2019, 166, F66-F73.

(22) Hansen, J. N.; Prats, H.; Toudahl, K. K.; Mørch Secher, N.; Chan, K.; Kibsgaard, J.; Chorkendorff, I. Is There Anything Better than Pt for HER? ACS Energy Letters 2021, 1175-1180.

(23) Chandler, K. G.; Genders, D. J.; Pletcher, D. Electrodes Based on Noble Metals. Platinum Metals Rev. 1997, 41, 54-63. 
(24) Hayfield, P. Development of the Noble Metal / Oxide Coated Titanium Electrode. Platinum Metals Review 1998, 42, 27-33.

(25) Kongkanand, A.; Gu, W.; Mathias, M. F. Fuel Cells and Hydrogen Production. Fuel Cells and Hydrogen Production 2019,

(26) Ferreira, P. J.; la O’, G. J.; Shao-Horn, Y.; Morgan, D.; Makharia, R.; Kocha, S.; Gasteiger, H. A. Instability of PtC Electrocatalysts in Proton Exchange Membrane Fuel Cells. Journal of The Electrochemical Society 2005, 152, A2256.

(27) Mom, R.; Frevel, L.; Velasco-Vélez, J. J.; Plodinec, M.; Knop-Gericke, A.; Schlögl, R. The Oxidation of Platinum under Wet Conditions Observed by Electrochemical X-ray Photoelectron Spectroscopy. Journal of the American Chemical Society 2019, 141, 6537-6544.

(28) Tang, L.; Han, B.; Persson, K.; Friesen, C.; He, T.; Sieradzki, K.; Ceder, G. Electrochemical stability of nanometer-scale Pt particles in acidic environments. Journal of the American Chemical Society 2010, 132, 596-600.

(29) Jacobse, L.; Rost, M. J.; Koper, M. T. Atomic-Scale Identification of the Electrochemical Roughening of Platinum. ACS Central Science 2019, 5, 1920-1928.

(30) Yang, H.; Tang, Y.; Zou, S. Electrochemical removal of surfactants from Pt nanocubes. Electrochemistry Communications 2014, 38, 134-137.

(31) Cherevko, S.; Zeradjanin, A. R.; Keeley, G. P.; Mayrhofer, K. J. J. A Comparative Study on Gold and Platinum Dissolution in Acidic and Alkaline Media. Journal of The Electrochemical Society 2014, 161, H822-H830.

(32) Wei, C.; Rao, R. R.; Peng, J.; Huang, B.; Stephens, I. E.; Risch, M.; Xu, Z. J.; Shao-Horn, Y. Recommended Practices and Benchmark Activity for Hydrogen and Oxygen Electrocatalysis in Water Splitting and Fuel Cells. Advanced Materials 2019, 31. 
(33) Deng, X.; Galli, F.; Koper, M. T. In Situ Electrochemical AFM Imaging of a Pt Electrode in Sulfuric Acid under Potential Cycling Conditions. Journal of the American Chemical Society 2018, 140, 13285-13291.

(34) Topalov, A. A.; Katsounaros, I.; Auinger, M.; Cherevko, S.; Meier, J. C.; Klemm, S. O.; Mayrhofer, K. J. Dissolution of platinum: Limits for the deployment of electrochemical energy conversion? Angewandte Chemie - International Edition 2012, 51, 12613-12615.

(35) Solla-Gullón, J.; Montiel, V.; Aldaz, A.; Clavilier, J. Electrochemical characterization of platinum nanoparticles prepared by microemulsion: How to clean them without loss of crystalline surface structure. Journal of Electroanalytical Chemistry 2000, 491, 69-77.

(36) Climent, V.; Feliu, J. M. Thirty years of platinum single crystal electrochemistry. Journal of Solid State Electrochemistry 2011, 15, 1297-1315.

(37) Mundschau, M.; Vanselow, R. Difficulties in the detection of surface impurities on platinum using Auger electron spectroscopy. Surface Science 1985, 157, 87-98.

(38) Salmeron, M.; Brewer, L.; Somorjai, G. The Structure and Stability of Surface Platinum Oxide and of Oxides of Other Noble Metals. Surface Science 1981, 112, 207-228.

(39) Hodnik, N.; Cherevko, S. Spot the difference at the nanoscale: identical location electron microscopy in electrocatalysis. Current Opinion in Electrochemistry 2019, 15, 73-82.

(40) Lafforgue, C.; Chatenet, M.; Dubau, L.; Dekel, D. R. Accelerated Stress Test of Pt/C Nanoparticles in an Interface with an Anion-Exchange Membrane - An Identical-Location Transmission Electron Microscopy Study. ACS Catalysis 2018, 8, 1278-1286.

(41) Ahmadi, T. S.; Wang, Z. L.; Green, T. C.; Henglein, A.; El-sayed, M. A. Shape-Controlled Synthesis of Colloidal Platinum Nanoparticles. Science 1996, 272, 1924.

(42) Mehrotra, U. S.; Agrawal, M. C.; Mushran, S. P. Reduction of hexachloroplatinate by ascorbic acid. Journal of Inorganic and Nuclear Chemistry 1970, 32, 2325-2329. 
(43) Vidal-Iglesias, F. J.; Arán-Ais, R. M.; Solla-Gullón, J.; Herrero, E.; Feliu, J. M. Electrochemical characterization of shape-controlled Pt nanoparticles in different supporting electrolytes. ACS Catalysis 2012, 2, 901-910.

(44) Parapat, R. Y.; Wijaya, M.; Schwarze, M.; Selve, S.; Willinger, M.; Schomäcker, R. Particle shape optimization by changing from an isotropic to an anisotropic nanostructure: Preparation of highly active and stable supported Pt catalysts in microemulsions. Nanoscale 2013, 5, 796805.

(45) Yang, M.; Xia, J. J. Preparation and characterization of platinum nanorods using ascorbic acid as the reducing agent. Advanced Materials Research 2013, 774-776, 577-580.

(46) Senapati, S.; Das, S. P.; Patnaik, A. K. Kinetics and mechanism of oxidation of L-Ascorbic acid by $\mathrm{Pt}(\mathrm{IV})(\mathrm{aq})$ in aqueous hydrochloric acid medium. Advances in Physical Chemistry 2012, 2012, 2-7.

(47) Hoefelmeyer, J. D.; Niesz, K.; Somorjai, G. A.; Tilley, T. D. Radial anisotropic growth of rhodium nanoparticles. Nano Letters $\mathbf{2 0 0 5}$, 5, 435-438.

(48) Song, Y.; Yang, Y.; Medforth, C. J.; Pereira, E.; Singh, A. K.; Xu, H.; Jiang, Y.; Brinker, C. J.; Van Swol, F.; Shelnutt, J. A. Controlled Synthesis of 2-D and 3-D Dendritic Platinum Nanostructures. Journal of the American Chemical Society 2004, 126, 635-645.

(49) Si, F.; Ma, L.; Liu, C.; Zhang, X.; Xing, W. The role of anisotropic structure and its aspect ratio: High-loading carbon nanospheres supported Pt nanowires with high performance toward methanol electrooxidation. RSC Advances 2012, 2, 401-403.

(50) Yang, T. H.; Shi, Y.; Janssen, A.; Xia, Y. Surface Capping Agents and Their Roles in ShapeControlled Synthesis of Colloidal Metal Nanocrystals. Angewandte Chemie - International Edition 2020, 59, 15378-15401. 
(51) Petroski, J. M.; Wang, Z. L.; Green, T. C.; El-Sayed, M. A. Kinetically controlled growth and shape formation mechanism of platinum nanoparticles. Journal of Physical Chemistry B 1998, 102, 3316-3320.

(52) Shiraishi, Y.; Toshima, N. Oxidation of ethylene catalyzed by colloidal dispersions of poly(sodium acrylate)-protected silver nanoclusters. Colloids and Surfaces A: Physicochemical and Engineering Aspects 2000, 169, 59-66.

(53) Solla-Gullón, J.; Vidal-Iglesias, F. J.; Rodríguez, P.; Herrero, E.; Feliu, J. M.; Clavilier, J.; Aldaz, A. In situ surface characterization of preferentially oriented platinum nanoparticles by using electrochemical structure sensitive adsorption reactions. Journal of Physical Chemistry B 2004, 108, 13573-13575.

(54) Solla-Gullón, J.; Rodríguez, P.; Herrero, E.; Aldaz, A.; Feliu, J. M. Surface characterization of platinum electrodes. Physical Chemistry Chemical Physics 2008, 10, 1359-1373.

(55) Henglein, A.; Ershov, B. G.; Malow, M. Absorption spectrum and some chemical reactions of colloidal platinum in aqueous solution. Journal of Physical Chemistry 1995, 99, 1412914136.

(56) Russell, A. E. Electrocatalysis: Theory and experiment at the interface. Physical Chemistry Chemical Physics 2008, 10, 3607-3608.

(57) Herrero, E.; Feliu, J. M.; Aldaz, A. CO monolayer oxidation on semi-spherical and preferentially oriented ( 1000$)$ and ( $\left.\begin{array}{lll}1 & 1 & 1\end{array}\right)$ platinum nanoparticles. 2006, 8, 189-194.

(58) Tong, K.; Song, X.; Xiao, G.; Yu, J. Colloidal processing of $\operatorname{Mg}(\mathrm{OH}) 2$ aqueous suspensions using sodium polyacrylate as dispersant. Industrial and Engineering Chemistry Research 2014, 53, 4755-4762.

(59) Buyse, K.; Gernon, M. D.; Matton, R.; Van de Woestijne, E. The benefits of alkanolamines over caustic: For pH neutralization of latex paint. PCI-Paint and Coatings Industry 2013, 29. 
(60) Kirwan, L. J.; Fawell, P. D.; Van Bronswijk, W. An in situ FTIR-ATR study of polyacrylate adsorbed onto hematite at high pH and high ionic strength. Langmuir 2004, 20, 4093-4100.

(61) Taylor, J. J.; Sigmund, W. M. Adsorption of sodium polyacrylate in high solids loading calcium carbonate slurries. Journal of Colloid and Interface Science 2010, 341, 298-302.

(62) Grabowska, B.; Holtzer, M. Structural examination of the cross-linking reaction mechanism of polyacrylate binding agents. Archives of Metallurgy and Materials 2009, 54, 427-437.

(63) Sheng, W.; Zhuang, Z.; Gao, M.; Zheng, J.; Chen, J. G.; Yan, Y. Correlating hydrogen oxidation and evolution activity on platinum at different $\mathrm{pH}$ with measured hydrogen binding energy. Nature Communications 2015, 6 .

(64) Yadav, A. P.; Okayasu, T.; Sugawara, Y.; Nishikata, A.; Tsuru, T. Effects of pH on Dissolution and Surface Area Loss of Platinum Due to Potential Cycling. 2012, 159, 190-194.

(65) Komanicky, V.; Chang, K. C.; Menzel, A.; Markovic, N. M.; You, H.; Wang, X.; Myers, D. Stability and Dissolution of Platinum Surfaces in Perchloric Acid. Journal of The Electrochemical Society 2006, 153, B446.

(66) Lopes, P. P.; Tripkovic, D.; Martins, P. F.; Strmcnik, D.; Ticianelli, E. A.; Stamenkovic, V. R.; Markovic, N. M. Dynamics of electrochemical Pt dissolution at atomic and molecular levels. Journal of Electroanalytical Chemistry 2018, 819, 123-129.

(67) Ota, K. I.; Nishigori, S.; Kamiya, N. Dissolution of platinum anodes in sulfuric acid solution. Journal of Electroanalytical Chemistry 1988, 257, 205-215.

(68) Lopes, P. P. et al. Eliminating dissolution of platinum-based electrocatalysts at the atomic scale. Nature Materials 2020, 19, 1207-1214.

(69) Pavlov, V. I.; Gerasimova, E. V.; Zolotukhina, E. V.; Don, G. M.; Dobrovolsky, Y. A.; Yaroslavtsev, A. B. Degradation of Pt/C electrocatalysts having different morphology in lowtemperature PEM fuel cells. Nanotechnologies in Russia 2016, 11, 743-750. 
(70) Rasouli, S.; Myers, D.; Kariuki, N.; Higashida, K.; Nakashima, N.; Ferreira, P. Electrochemical Degradation of Pt-Ni Nanocatalysts: An Identical Location Aberration-Corrected Scanning Transmission Electron Microscopy Study. Nano Letters 2019, 19, 46-53.

(71) Pavlišič, A.; Jovanovič, P.; Šelih, V. S.; Šala, M.; Hodnik, N.; Gaberšček, M. Platinum Dissolution and Redeposition from Pt/C Fuel Cell Electrocatalyst at Potential Cycling. Journal of The Electrochemical Society 2018, 165, F3161-F3165.

(72) Scheijen, F. J.; Beltramo, G. L.; Hoeppener, S.; Housmans, T. H.; Koper, M. T. The electrooxidation of small organic molecules on platinum nanoparticles supported on gold: Influence of platinum deposition procedure. Journal of Solid State Electrochemistry 2008, 12, 483-495.

(73) Ramaker, D. E.; Gatewood, D.; Korovina, A.; Garsany, Y.; Swider-Lyons, K. E. Resolving sulfur oxidation and removal from Pt and Pt3Co electrocatalysts using in situ X-ray absorption spectroscopy. Journal of Physical Chemistry C 2010, 114, 11886-11897.

(74) Quijada, C.; Rodes, A.; Vázquez, J. L.; Pérez, J. M.; Aldaz, A. Electrochemical behaviour of aqueous SO2 at Pt electrodes in acidic medium. A voltammetric and in situ Fourier transform IR study Part I. Oxidation of SO2 on Pt electrodes with sulphur-oxygen adsorbed species. Journal of Electroanalytical Chemistry 1995, 394, 217-227.

(75) Durst, J.; Simon, C.; Hasché, F.; Gasteiger, H. A. Hydrogen Oxidation and Evolution Reaction Kinetics on Carbon Supported Pt, Ir, Rh, and Pd Electrocatalysts in Acidic Media. Journal of The Electrochemical Society 2015, 162, F190-F203.

(76) Zheng, J.; Yan, Y.; Xu, B. Correcting the Hydrogen Diffusion Limitation in Rotating Disk Electrode Measurements of Hydrogen Evolution Reaction Kinetics. Journal of The Electrochemical Society 2015, 162, F1470-F1481.

(77) Wang, H.; Abruña, H. D. Rh and Rh Alloy Nanoparticles as Highly Active H2 Oxidation Catalysts for Alkaline Fuel Cells. ACS Catalysis 2019, 9, 5057-5062. 
(78) Wang, H.; Abruña, H. D. Designing Synergistic Electrocatalysts for H2Oxidation and Evolution Reactions in Alkaline Media. Journal of Physical Chemistry C 2021, 125, 7188-7203.

(79) Subbaraman, R.; Tripkovic, D.; Strmcnik, D.; Chang, K. C.; Uchimura, M.; Paulikas, a. P.; Stamenkovic, V.; Markovic, N. M. |vac ) given an expected mean number of events $\mathrm{m} 0=\mathrm{d}$ 2 Np. Science 2011, 334, 1256-1260.

(80) Hagyard, T.; Williams, J. R. Potential of aluminium in aqueous chloride solutions. Part 1. Transactions of the Faraday Society 1961, 57, 2288-2294. 